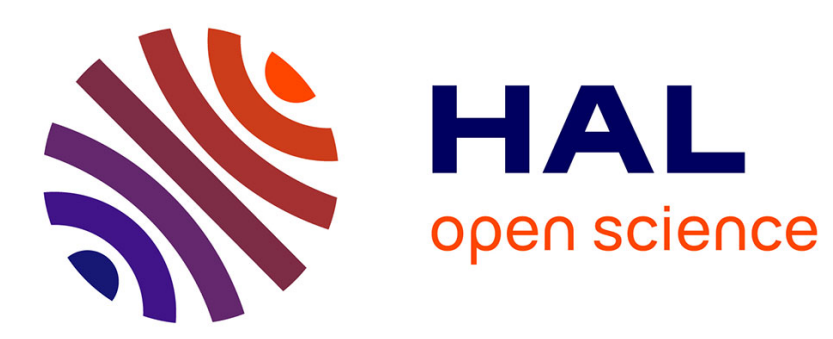

\title{
AMANDA V3: Toward a Common Workspace between Air Traffic Controllers
}

David Annebicque, Serge Debernard, Thierry Poulain, Igor Crevits

\section{To cite this version:}

David Annebicque, Serge Debernard, Thierry Poulain, Igor Crevits. AMANDA V3: Toward a Common Workspace between Air Traffic Controllers. First International Conference on Advances in Computer-Human Interaction, Feb 2008, Sainte Luce, France. pp.212-217. hal-02112923

\section{HAL Id: hal-02112923 \\ https://hal.science/hal-02112923}

Submitted on 27 Apr 2019

HAL is a multi-disciplinary open access archive for the deposit and dissemination of scientific research documents, whether they are published or not. The documents may come from teaching and research institutions in France or abroad, or from public or private research centers.
L'archive ouverte pluridisciplinaire HAL, est destinée au dépôt et à la diffusion de documents scientifiques de niveau recherche, publiés ou non, émanant des établissements d'enseignement et de recherche français ou étrangers, des laboratoires publics ou privés. 


\title{
AMANDA V3: Toward a common workspace between air traffic controllers
}

\author{
David Annebicque, Serge Debernard, Thierry Poulain, Igor Crévits
}

\begin{abstract}
This paper presents the different tools developed in the LAMIH, in optics to assist air traffic controllers in their tasks, to decrease their workloads, and to enable them to support the ceaseless increase of the traffic. Common philosophy to all these tools is to preserve the controllers in the loop: we do not try to develop tools entirely automatic. The platform AMANDA V2 made it possible to set up and to evaluate a common workspace, which allows the two controllers of a sector to cooperate and to share the same representation of their traffic and conflicts. This space maintains common situation awareness. This tool was very appreciated by professional controllers and we now wish to extend this principle to the co-operation between two planning controllers of two adjacent sectors. It is what we present in this paper which begins with a presentation of the ATC then a point on the platforms of the laboratory and particularly AMANDA V2, to conclude with the objectives of AMANDA V3.
\end{abstract}

Index Terms - Air Traffic Control, Human-Machine cooperation, dynamic allocation of function, support tool, common workspace

\section{INTRODUCTION}

$\mathrm{T}$ HE Air Traffic Control (ATC) is a domain where the complete automation is difficult to conceive. First of all, in a technical point of view, a complete automation would impose equipment on the whole of the aircraft, what would be excessively expensive, and would call upon technologies under development (e.g. datalink), not even developed yet (conflict detection, reliable forecast of trajectory, weather forecasting...). Then it is also difficult to conceive from a human point of view: pilots and controllers remain at the present time guarantors of safety.

However, it is necessary to help controllers in their tasks. Indeed, the air traffic does not stop to increase (5\% per year) and human capacities begin to be reached. To decrease their load of traffic per division of the zones which they control (sectors) does not seem possible, because the reduction in their

Manuscript received September 19, 2007. This work is supported by the French Department of Aviation (DGAC/SDER/DTI).

David Annebicque is a $\mathrm{PhD}$ student at the LAMIH, University of Valenciennes, Le Mont Houy, F 59313 Valenciennes Cedex 9, France (corresponding author: +33 (0) 3275113 73; fax: +33 (0) 3275113 16; email: david.annebicque@univ-valenciennes.fr).

Serge Debernard, is a Professor at the LAMIH, University of Valenciennes, (e-mail: serge.debernard@univ-valenciennes.fr).

Thierry Poulain is a research engineer at the LAMIH, University of Valenciennes, (e-mail: thierry.poulain@univ-valenciennes.fr).

Igor Crévits is a Associate Professor at the LAMIH, University of Valenciennes, (e-mail: igor.crevits@univ-valenciennes.fr). surface would not give enough time to anticipate and correct the problems, i.e. the air conflicts. It is thus necessary to find a compromise between a full automatic system and a purely manual system. With our opinion, this compromise can be reached by developing new co-operative assistance tools, i.e. the controller preserves the control on the whole of the process, and the tools come only to assist it in its task.

The LAMIH (French acronym for Laboratory of Automation, Mechanics and Human Industrial Computer), and more particularly the HMS team (Human-Machine System) works in this perspective since many years. In partnership with the SDER/DTI (French acronym for Direction Study and research / Direction of the Technique and the Innovation) the laboratory designs tools able to help controllers. The objective is to reduce the controllers' workload - to help him "to absorb" the ceaseless increase of the traffic - without "to take off" the controller of the control loop: especially, new systems must be conceived in order to maintain a "good" situation awareness which allows controllers to take again the hand in the event of failure of a system. This partnership led to the development of several experimental platforms: SPECTRA, AMANDA (Automated MAN-machine Delegation of Action), which allowed to test various tools with professional controllers. In particular, the introduction of a Common Workspace between the controllers of a sector (executive \& planning controllers) and a assistance tool - which is able to integrate human strategies for the resolution of a conflict - permit to various agents to share the same representation of the air conflicts, that supports human anticipation and the maintenance of the situation awareness [1, 2, 3]. This Common Workspace developed first of all on the platform AMANDA V2 and this for only one sector, now will be extended to the co-operation between planning controllers of adjacent sectors: these are the work which is presented in this paper.

Before presenting this new space, we will point out the functioning of the ATC, while insisting on the co-operations between adjacent sectors, and the problems that that implies in the more or less long term (overload of the operators). Then, we will point out the bases of the platform AMANDA V2 and to show the need for extending the concept to several sectors. Finally in a last part, we will present the objectives of AMANDA V3, and the first solutions considered.

\section{Presentation of AiR Traffic Control}

\section{A. Objectives and organization of Air Traffic Control}

\section{1) Objectives}

The principal objective of ATC is to guarantee an optimal safety to the whole of the aircraft. To guarantee an optimal 
safety consists in anticipating and avoiding any collision between aircraft, or between an aircraft and the relief. Airtraffic controllers also have the role of preventing that a aircraft crosses prohibited zones (military space) or dangerous (stormy zone for example). They take care of the respect of the flight plans. In the event of conflict (two or more aircraft are not separated by a minimal distance on the horizontal plan or vertical level) the controllers must modify the trajectories in order to restore these distances. They endeavor to do that, while guaranteeing minimal delays and costs.

\section{2) Organisation}

The ATC is organized in three levels. A first level called "Airport control" manages the aircraft landing, taking off, and forwarding on airport. A second level called "Approach and terminal control" has in responsibility of prepare the aircraft before their landing on the airports. It is in this space that the aircraft are controlled and sequenced for the landing. Finally, the last level is "En-route control". It has the management of the superior level, and manages the flights of their starting airports to the terminal zones. It is on this level that our study is interested.

En-route control occupies the major part of the airspace, and has the responsibility of the major part of the time of flight. The French sky is divided into 5 centers of En-route control, called CRNA (Regional Center of Air Navigation). Each center is then divided into sectors, sectors which can be regrouped according to the load of traffic. Each sector has an organization in binomial, with an "executive controller" or "tactical controller" (EC.), and a "planning controller" (PC). The first one has in charge the detection and the resolution of the conflicts, communicates with the pilots, and takes care of the respect of the trajectories. The second one has a role of coordination with the other adjacent sectors. He deals with accepting the aircraft in his sector, and prepares the aircraft leaving for the other sectors. It is mainly brought to communicate, and to cooperate with its counterparts of the adjacent sectors.

\section{B. Limits of present organization}

The principal limitation of the current system of the ATC is the risk of overload of the controllers, in particular for the EC. To avoid these overloads which would not allow to maintain an optimal level of safety, different solutions are adopted, like the planning of the flights and the regulation at the beginning of the airports, or the coordination between sectors which permits to reduce the complexity of the air conflicts, to even prevent that these conflicts take place really.

This coordination is carried out between planning controllers of adjacent sectors and are carried out by telephone. A change of coordination for a flight is in fact a negotiation between PCs: a request for changing a flight level or a trajectory must then be checked in order to evaluate the consequences of these modifications on the traffic. Nevertheless, these negotiations can take time and require synchronization between the PCs' activities, which in the long term can raise difficulties in the event of high traffic.

It can thus be interesting to help them, to facilitate the negotiations to make it possible in the cases of overload to conclude their tasks all the same. That's why we propose to develop a Common Workspace able to assist PCs in their tasks of negotiations. We first of all present the project AMANDA V2 in which a Common Workspace between one PC, one EC and assistance tool to the resolution of air conflicts was developed and evaluated with professional controllers.

\section{AMANDA V2 FOR ONE SECTOR}

\section{A. Philosophy}

The platform AMANDA is the continuity of other projects developed within the laboratory since many years (SPECTRA $\mathrm{V} 1 \& \mathrm{~V} 2[4,5,6])$. These projects are placed in perspective to preserve the human in the control loop, and thus not to entirely automate the task of the ATC to avoid the loss of Situation Awareness (SA) and in a long-term the degradation of their competencies.

SPECTRA V1 \& V2 made it possible to evaluate in experiments the concept of co-operation between controllers and an autonomous system of assistance to the resolution of conflict called SAINTEX. The mode of co-operation tested consisted then in a dynamic distribution of tasks.

In the first version of SPECTRA, only the EC cooperate with the resolution assistance system which was conceived to solve only certain binary conflicts. SAINTEX could thus manage only part of the traffic and the conflicts, the others always having to be managed by EC. Two approaches were tested: a first known as "explicit" where EC chose which him or the system was to solve the conflict, and a second known as "implicit" where the system ensured the distribution according to following criteria's: the controller's workload, and the capacity of the system to solve the conflict.

Even if the implicit mode gave the best results, EC preferred the explicit distribution, avoiding thus certain decisional conflicts. This first version thus made it possible to emphasize two important points:

- An operator cannot simultaneously carry out a strategic task (distribution) and a tactical task (resolution of conflicts) without reducing in a detrimental way the performances obtained.

- So that a controller can continue to manage the whole of the traffic, it is necessary to define the tasks well that the resolution assistance system to in charge, and that the division or the decomposition of the conflicts is coherent with the representation of the controllers.

A second version of SPECTRA $[4,5]$ was then developed to try to answer to the problems raised in the first version. In this version, the implicit mode was given up and, in order to avoid charging EC, it is the PC who had in charge the management of the distribution of the conflicts, the EC ensuring only the resolution of conflict. This approach led to a better performance (better planning, better co-operation). On the other hand EC tended to leave the supervision of the traffic with PC (in charge of the distribution, and thus of the detection of the conflicts), which leads to two new problems in the human-machine co-operation:

- A complacency phenomenon where the shared supervision too often results in a division of the environment without mutual control [7].

- A limitation of the resolution assistance system, which induced many counter-order on behalf of EC. It proves that the really binary conflicts are rare, and 
that it is advisable to take into account a certain number of "contextual" (not directly in conflict) aircraft in the resolution, for a better solution [8].

AMANDA is born thus following these two problems. In this project, a new assistance tool has been designed, STAR (French acronym for Tactical System for Resolution Aid [9]) in order to answer the second problem and the limitations of SAINTEX. The controller will give to STAR a strategy for resolving the problem, and then STAR will inform controller of possible interfering aircraft and calculate a new trajectory for avoiding the initial conflict. Now we will see how AMANDA works, and integrates the human controller in the loop.

\section{B. Principles on one sector}

The platform AMANDA V2 is composed of two principal functions [10]: STAR [9] and a Common Workspace [11, 12, $13,14]$. STAR brings a help to the controllers for the calculation of trajectory, and allows a delegation of the conflict resolutions to the machine. The Common Workspace allows a communication and a sharing between all the participants (PC, EC, and STAR). Let us see now more in details these two functionalities.

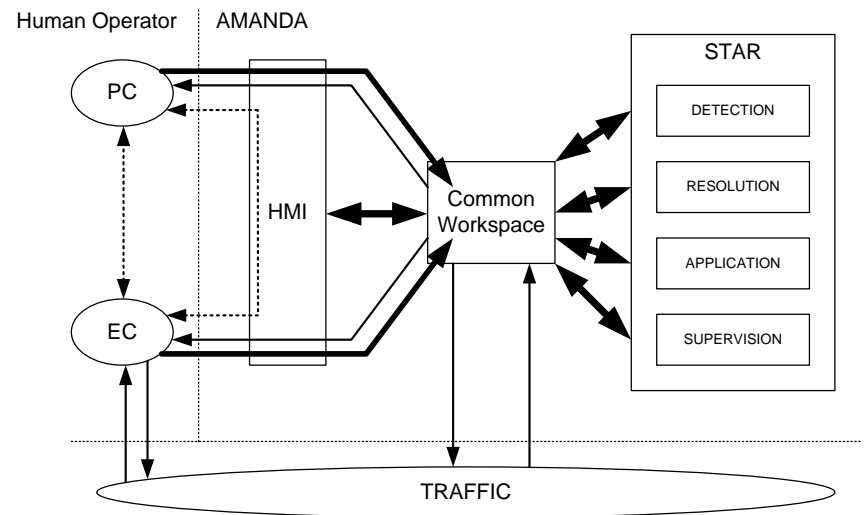

Figure 1 Diagram of the actual platform AMANDA V2

\section{1) Common Workspace}

The common workspace has for objective to provide a richer environment of work, by confronting the ideas, the thought of the different agents. In our case we have two controllers (human agents) and STAR (artificial agent, machine). Each agent is thus brought to complete, to inform this common workspace, according to its competencies (know-how) but also of his role in the process. All the agents can take note constantly of information which is recorded in this workspace, in order to carry out their tasks, or to control and check those of the other agents.

This Common Workspace thus makes it possible mainly to maintain a common situation awareness [1, 2, 3] between the two controllers, to share their representation of the problems (in sense of air conflicts, loss of separation) to solve or to supervise. The controllers have in responsibility of maintain up to date this space, in order to on the one hand to preserve a coherent "picture" of the situation, and on the other hand to inform the platform, and mainly STAR, with conflicts which the controllers detect. This task of conflict detection, annotation and selection of the aircraft included in a problem, already exists in current control, which is materialized by bringing together the strips, by a distinctive sign on the aircraft of the same conflict, and a communication between the controllers.

The common workspace contains two principal functions: the first one is a list of the problems created by the controllers, and the second is a radar view, which makes it possible to the controllers to choose a strategy of resolution in order to use STAR. This view is called "problem resolution view". The first view, or "cluster view" thus gathers a list of problem, each problem, or cluster, gathers several information.

- The list of the aircraft implied. It is the list of the aircraft which the controllers added to the problem, and which are potentially in conflict, or to take into account for the resolution of the problem. This list can also contain the aircraft that STAR detected as in conflict with one of the aircraft chosen by the controllers.

- The state of the problem. This information is given by the system, and STAR. It indicates how STAR perceives the problem. There are six possible states for a problem: detected (the conflict is well detected), treated (controller provided information necessary to solve the problem), must be delegate (STAR can find a trajectory which solves the problem), delegated (STAR deals with applying the trajectory), not solvable (STAR cannot solve the problem).

- The list of the directives provides by the controllers which correspond to strategies of avoidance such as for example: AFR432 turn behind BAW657.

- "Answers" of STAR. It is mainly about the minimal distance from separation between two aircraft (binary conflict), before OR after the implementation of a strategy of resolution. STAR indicates also the distance from separation which results from the application of a directive or a differed order, in order to inform the controller on "the effectiveness" of his/her solution.

The second view, the "problem resolution view", is accessible from one problem or cluster from the previous list ("cluster view"). It is based on a traditional radar view. This view emphasizes the aircraft of the problem, and those which could intervene in the resolution (on the same level for example). The other flights are posted "set back" in order to not disturb, or obstruct the view of the controllers. The trajectories of the aircraft included in the problem are also displayed with different colors. From this view the controllers can: add or remove aircraft in the cluster, "feed" or communicate with STAR and choose differed orders or directives, and finally delegate the conflict resolution to the machine. Nevertheless, the controller always keeps the possibility to take back a delegated conflict.

\section{2) STAR}

STAR is a tool which ensures a certain number of functions in order to assist the controllers in their work and in particular the task of conflict resolution. The principle used with STAR is to help the controllers by delegating part of their tasks to an automatic system, by considering that the system is sufficiently qualified and reliable to discharge the controllers from part of their activities. The objective is not to automate 
air traffic control: STAR does not have competencies for defining a strategy of resolution, and it is thus leaned on the controller who remains in the center of the loop. STAR, starting from a strategy of resolution (directive) provided by the controller, will determine a new trajectory answering this directive and ensuring the separation of the flights in conflict. The controller has then the possibility of delegating the application of the trajectory to the system, which will be given the responsibility to communicate the instructions to the aircraft.

During the calculation of the trajectory, STAR checks that this one does not create a new conflict. If however STAR does not find trajectories which do not produce a new conflict, it informs the controllers of them, so that they find a solution. This new conflict can come owing to the fact that the controllers forgot in the creation of the problem an aircraft to be taken into account, or whereas they informed the problem well, but which they did not choose yet of solution for the interfering aircraft.

STAR ensures 3 functions:

- STAR supervises the conformity of the aircraft trajectories relatively to the flight plans, or the trajectories defined by the controllers or the system. This function also informs on the respect of the distances (minimal separation), calculated and updated in real time.

- The delegation of a differed order which the controller defined, and that STAR will apply. A differed order is completely specified but it must be applied to a precise moment. For example "aircraft X must climb on level 300 at this time". STAR will check that this order does not produce a conflict, and if the trajectory is then validated the controller can choose to delegate it to the system, and this one will deal with the implementation of the trajectory. The controller has also the possibility of defining a succession of differed order on a flight, and the system will deal with successively applying them in chronological order.

- Finally the delegation of a directive on a flight. A directive is a strategy of resolution for a binary conflict. A directive is an incomplete order, and not defines precisely the trajectory, but an "idea" of the trajectory. To create a directive it is necessary to define a target which will undergo the deviation, the new trajectory, and a privileged flight (which it is necessary to avoid), and a strategy (for example: To pass in front of, turn behind). Thus the directive: "Aircraft A TURN_BEHIND aircraft B" means that aircraft $\mathrm{A}$ is the conflicting aircraft, the aircraft $\mathrm{B}$ the privileged, and that aircraft $A$ will avoid the aircraft B while turning behind it. STAR first of all will calculate ALL the trajectories which answer this strategy, then characterize them starting from various criteria (safety, cost, number of deviations...). Finally, STAR will eliminate the whole of the trajectories creating new conflicts, and then propose only one and single trajectory to the controllers. They will have then the possibility of delegating this resolution to the system.

\section{Some results of experimentations}

This version of AMANDA was tested with professional controllers of Bordeaux. We wanted to test, and evaluate the tools placed at the disposal of the controllers (STAR, Common Workspace) and to study the best distribution of the tasks between the two controllers (PC and EC). We tested three situations:

- Situation A (Situation of reference): In this situation, the clusters are only created by PC. STAR can not be used, and the resolutions remain with the responsibility of EC.

- Situation B (Situation PC - STAR): It is the situation integrating the whole of the assistances. PC creates the clusters and feeds them with differed orders and directives. EC can delegate to STAR the clusters. Nevertheless EC can modify the differed orders and directives if it's necessary.

- Situation C (Situation EC - STAR): As previously, all tools are available but PC ensures only the creation of the clusters. EC supplements the clusters with differed orders and directive and then can delegate them to STAR.

Four binomial of controllers has tested the three situations according to three different scenarios of traffic, in permuting the order of handover to avoid any effects of order. Subjective data were collected (questionnaires [14]), as well as objective data (backup of handover). The controllers' workload was evaluated with the TLX method.

The analysis of the workload highlights that the situation $\mathrm{C}$ is worst, because it increases the workload of EC considerably. The situations with all tools (B and C) generate a rise of the workload due to the use of the interfaces, but this rise does not seem too much hamper the controllers in situation B relatively to the profits brought (delegation of the flights...).

The rest of the study allowed evaluating the interfaces and the tools. The questionnaires confirm the analysis of the workload, and show that the situation B seems to be most interesting. The controllers although more solicited in the situations with assistance, estimated that these assistances decreased their workload. The controllers find that STAR brings benefit to the realization of their tasks, and finds an undeniable help in the Common Workspace. Particularly in situation B, the Common Workspace supports human cooperative activities and the controllers almost did not speak during the scenarios.

The analysis of the objective data, confirms these results. The controllers created clusters in $93 \%$ of the cases where there was conflict. For $75 \%$ of these clusters, there was a directive or a differed order introduced by the controllers and $63 \%$ from these directives or orders were delegated to the system.

This analysis allowed noting a certain number of points to be improved or to take into account. We will see them in the following part. 


\section{Problems and limits}

Following to the experiments some problems and limitations appeared. Generally the platform and philosophy were very well accommodated by the controllers. But they reproach lack of flexibility and a lack of intuitiveness of the interface. For this point no precise ergonomic study was undertaken.

The fact that the platform is focused only on one sector, and that thus the whole of the communications with the adjacent sectors are not supported, have favored a very great anticipation of the controllers' activities, with the assumption of responsibility of aircraft well before their sector, which reduced the number of conflict and decreased the load of the sectors. This lack of the adjacent sectors also distorted uncertainties on the conditions of entry of the aircraft which could not be modified upstream. That also appreciably reduced the task of the PC who was not to negotiate the entries and the exits of the aircraft.

The second point relates to STAR which on the one hand showed lacks for the conflicts with more than two aircraft, or when the delegation was too late, providing "original" trajectories and on the other hand the controllers found that STAR turned a little too late, imposing to the aircraft angles or cap very strong, whereas it was not necessary. And for the handing-over on the road was too much close to the aircraft to avoid (respect with just of the standards of separation).

\section{AMANDA V3}

\section{A. Objectives}

The objectives of this new version of the platform AMANDA are on the one hand to correct the defects detected during the experiments, to take into account the remarks of the controllers, in particular for STAR, and on the other hand to integrate the adjacent sectors by providing, on the same principle, a common workspace for the PCs of adjacent sectors.

To introduce the adjacent sectors and to extend the principles of the common workspace to the co-operation between "distant" planning controllers present several interests:

- This new common workspace will facilitate the negotiations between the sectors, to make it possible to quickly visualize the flights concerned with the negotiation. Thus the workload, time necessary, and the risks of ambiguities should be reduced.

- This new common workspace will make it possible to share between the sectors, the modifications on the aircraft's trajectories, which should make it possible to reduce uncertainties on the positions and the entries conditions of a flight in a sector.

In parallel a specific study will be undertaken in order to improve STAR, by taking into account the aircraft which must change their flight level in a sector (unstable aircraft) and the concept of "interfering" aircraft (aircraft that the machine considers it necessary to take into account to solve a problem, and thus in many cases unstable aircraft). The addition of these "interfering" aircraft has constrained the controllers who did not regard them as awkward (bus which can climb/go down apart from the conflict). The controllers did not take them into account in their mental representation of the conflicts, and it will be necessary to think of systematically propose the interfering aircraft in the clusters.

\section{B. Principles of new "Common Workspace"}

This new Common Workspace will make it possible a controller (EC) to share easily and quickly information necessary to his/her task of coordination and co-operation with an adjacent sector and based on the same principle as for the current common workspace of AMANDA V2 which allows two controllers of the same position to share the same representation of the problems (clusters).

The "extended" Common Workspace will have to be closely dependent with the tools already available to the controllers, and particularly the existing common workspace. It is judicious to wonder if the two controllers of a position require to reach this new tool, or if it "is reserved" for PCs. And in this case the controllers will have to be able to browse quickly between the Common Workspace of their sector, and the "inter sector" Common Workspace.

This new Common Workspace will allow two distant controllers to immediately visualize information on the flight concerned by a negotiation (a change of the entry conditions in the following sector). This will bring a certain safety. All the actions will be "confirmed" by "writing" this on the Common Workspace in the aim to significantly reduce the necessary time to the realization of this task, to increase safety, and well to reduce the workload of the controllers.

Moreover this space creates a direct and permanent link between two planning controllers and can desynchronizes their activities. This will allow controller choosing the best moment to negotiate with its counterpart, and to be more efficient.

Finally, this link between sectors, this "extended" Common Workspace should reduce in considerable way uncertainties on the position of the aircraft in the entry of the sector. Currently the controllers work with strips (paper bands which present flight plan), but these strips are not updated automatically in real time. The controllers annotate them according to the negotiations. Moreover in AMANDA V2, the trajectories of the aircraft can be shown by simple click on the aircraft on the radar view, with the hours of passage to each beacon. This function was very appreciated by controllers, because allowing a very fast visualization. But as AMANDA V2 functions only on one sector of control, the problem of the changes of entry conditions of the aircraft was not managed. Thanks to the "extended" common workspace, the modifications on trajectories will be immediately transmitted to all the sectors and the visualization of the "new" roads will be always up to date. 


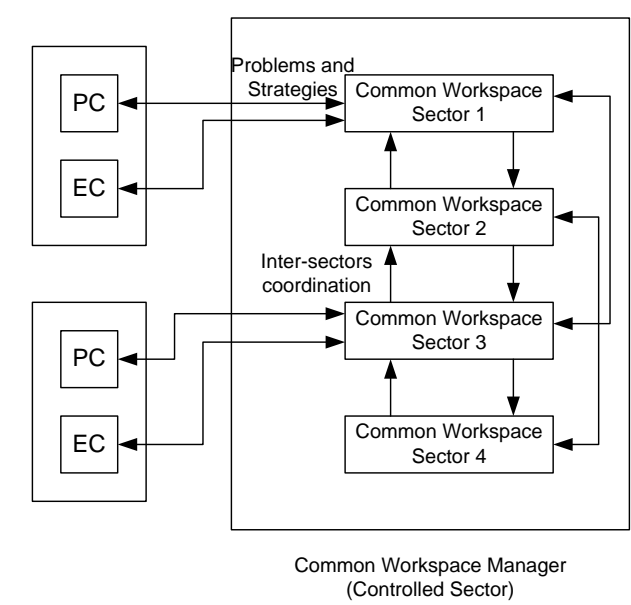

Figure 2 Diagram of the « extended » common workspace proposed by Guiost [14]

\section{CONCLUSION}

In this paper we started by presenting the ATC, and its limits. Then we made a reminder of various projects carried out within the laboratory in order to answer these different limitations, and to assist the controllers in the resolution of their tasks. The whole of these platforms have a common philosophy which consists in keeping, and to imply the controllers in the loop. In SPECTRA, a dynamic task allocation between controllers and an autonomous artificial agent has been tested: this solution shows its limits because generates decisional conflicts between agents. Following these projects, lesson was drawn, and new concepts are proposed in a new project called AMANDA. With this project the controller takes a more important place, and the artificial system (STAR) becomes assistance: the controller has the possibility to delegate certain tasks to the machine. An important tool is introduced: the Common Workspace which allows the two controllers and STAR "to communicate" and especially to keep common situation awareness by avoiding any decisional conflict between them. The platform AMANDA V2 assists the controllers on only one sector, while dealing with solving conflicts which the controllers delegated to him (voluntarily). The controllers "impose" to the machine the way for resolving a conflict (directive, differed order). This platform was the subject of experiments which confirmed our choices. Now we want to extend this principle to the cooperation between adjacent sectors. In AMANDA V3 all sectors will be equipped with a common workspace and the same tools as AMANDA V2 (modified and improved to answer the conclusions of the experiments). Then, by sharing information between these common work space, an extended common workspace will be designed, to assist and facilitate the negotiations, and the co-operation between two planning controllers.

\section{ACKNOWLEDGMENT}

We want to thank the DGAC/SDER/DTI for their support as well financial as human. We also thank all the personnel of the ATC, which by its availability and its collaboration made it possible to develop, and to evaluate the various platforms.

\section{REFERENCES}

[1] M.R. Endsley, "Toward a theory of situation awareness in dynamic systems", in Human Factors vol. 37, 1995, pp. 32-64.

[2] M.R. Endsley, D.B. Kaber, "Level of automation, effects on performance, situation awareness and workload in a dynamic control task", Ergonomics, Vol. 42(3), 1999, pp. 462-492.

[3] M.R. Endsley, "Automation and situation awareness », in R. Parasraman $\&$ M. Mouloua (Eds.), Automation and human performance: Theory and application, Mahwah, NJ: Lawrence Erlbaum Associates, 1996, pp. 163181.

[4] S. Debernard, "Contribution à la répartition dynamique des tâches entre opérateur et système automatisé : application au contrôle de trafic aérien", Ph.D. dissertation, LAMIH, Valenciennes Univ., Valenciennes, France, 1993.

[5] I. Crévits, "Répartition dynamique de tâches dans les procédés complexes : application au contrôle de trafic aérien", Ph.D. dissertation, LAMIH, Valenciennes Univ., Valenciennes, France, 1996.

[6] M.-P. Lemoine-Pacaux, "Coopération Hommes-Machines dans les procédés complexes", Ph.D. dissertation, LAMIH, Valenciennes Univ., Valenciennes, France, 1998.

[7] E.M., Roth, J.T. Malin, J.T., D.L. Schreckenghost, "Paradigms for intelligent interface design", in M. Helander, T.K. Landauer, \& P. Prabhu (Eds.), Handbook of human-computer interaction, Amsterdam: North-Holland, 1997, pp. 1177-1201.

[8] A. Bisseret, "Représentation et décision experte : psychologie cognitive de la décision chez les aiguilleurs du ciel", Octarés, Toulouse, France, 1995.

[9] S. Cathelain, "Contribution à la conception des systèmes coopératifs. Application au domaine du contrôle de trafic aérien”, Ph.D. dissertation, LAMIH, Valenciennes Univ., Valenciennes, France, 2005.

[10] S. Debernard, J.-M. Hoc, P. Denecker, "Projet AMANDA, rapport final de la deuxième phase", Centre d'Etude de la Navigation Aérienne CENA, Athis-Mons, RAPPORT LAMIH/CENA 96/C0007, 2001.

[11] M.-P. Pacaux-Lemoine, S. Debernard S., "Common work space for human-machine cooperation in air traffic control”, Elsevier, 2001.

[12] R. Bentley, T. Rodden, P. Sawyer, I. Sommerville, "An architecture for tailoring cooperative cooperative multi-user displays", presented in Conference proceeding of 4 th Computer -supported cooperative work, Toronto, Canada, 1992, pp. 187-194.

[13] Decortis, Pavard, "Communication et Coopération : de la théorie des actes du langage à l'approche éthnométhodologique", in Pavard \& al. (Ed.), Système coopératifs : de la modélisation à la conception, Octarès, toulouse, France, ISBN 2-906769-18-5.

[14] B. Guiost, "Coopération Homme-Machine soutenue par un espace de travail commun. Application au contrôle du trafic aérien", Ph.D. dissertation, LAMIH, Valenciennes Univ., Valenciennes, France, 2006. 